\title{
Effect of Prophylactic Vaccination with the Membrane-Bound Acid Phosphatase Gene of Leishmania mexicana in the Murine Model of Localized Cutaneous Leishmaniasis
}

\author{
María Angélica Burgos-Reyes, Lidia Baylón-Pacheco, Patricia Espíritu-Gordillo, \\ Silvia Galindo-Gómez, Víctor Tsutsumi, and José Luis Rosales-Encina $\mathbb{D}$ \\ Departamento de Infectómica y Patogénesis Molecular, Centro de Investigación y de Estudios Avanzados del Instituto \\ Politécnico Nacional, México City, Mexico \\ Correspondence should be addressed to José Luis Rosales-Encina; ros-tal@hotmail.com
}

Received 18 December 2020; Revised 20 March 2021; Accepted 30 March 2021; Published 10 April 2021

Academic Editor: Márcia Laurenti

Copyright (c) 2021 María Angélica Burgos-Reyes et al. This is an open access article distributed under the Creative Commons Attribution License, which permits unrestricted use, distribution, and reproduction in any medium, provided the original work is properly cited.

\begin{abstract}
Leishmaniasis is a disease caused by an intracellular protozoan parasite of the genus Leishmania. Current treatments for leishmaniasis are long, toxic, and expensive and are not available in some endemic regions. Attempts to develop an effective vaccine are feasible, but no vaccine is in active clinical use. In this study, the LmxMBA gene of Leishmania mexicana was selected as a possible vaccine candidate using the reverse vaccinology approach, and the prophylactic effect generated by DNA vaccination with this gene in a murine model of cutaneous leishmaniasis was evaluated. The results showed that prophylactic vaccination with pVAX1::LmxMBA significantly reduced the size of the lesion and the parasitic load on the footpad, compared to the control groups. At a histological level, a smaller number of parasites were evident in the dermis, as well as the absence of connective tissue damage. Mice immunized with plasmid pVAX1::LmxMBA induced immunity characterized by an increase in the IgG2a/IgG1 > 1 ratio and a higher rate of lymphocyte proliferation. In this study, immunization with the plasmid promoted an improvement in the macroscopic and microscopic clinical manifestations of the experimental infection by L. mexicana, with a $\mathrm{T}$ helper 1 response characterized by an IgG2a/IgG1 > 1 ratio and high lymphoproliferative response. These findings support immunization with the plasmid pVAX1::LmxMBA as a preventive strategy against cutaneous infection of $L$. mexicana.
\end{abstract}

\section{Introduction}

Leishmaniasis is an endemic disease in 97 countries and is considered a neglected tropical disease. A total of 1 billion people living in endemic areas are at risk of acquiring the disease, and it is estimated that there are at least 12 million cases of the various forms of leishmaniasis worldwide [1-3]. Cutaneous leishmaniasis (CL) caused by L. mexicana, L. amazonensis, L. major, and L. braziliensis is the most common form of leishmaniasis [4]. Visceral leishmaniasis (VL) caused by $L$. donovani and $L$. infantum is the most severe and fatal disease [5].

The immune cell response is essential in the control of Leishmania infection. The development of specific $\mathrm{T}$ helper 1 (Th1) response, based on the production of proinflamma- tory cytokines, such as interferon-gamma (IFN- $\gamma$ ), interleukin 12 (IL-12), and tumor necrosis factor-alpha (TNF- $\alpha$ ), can protect mammalian hosts from infection by the parasites. In contrast, a Th2 response supports the persistence of parasites and can cause an active disease [6,7]. Over the past years, studies have been developed to identify new candidates for the prevention of leishmaniasis and generate protective immunity [8-10]. However, there is still no effective prophylactic vaccination against human disease. First-generation vaccines with attenuated parasites concern potential reversion to virulence and raise a risk of reversion to clinical disease in immunocompromised individuals. On the other hand, second-generation vaccines might induce mainly humoral responses and are poorly immunogenic when administered without the association of adjuvants $[8,11,12]$. 
Another approach is the use of antigen-encoding plasmid DNA as an efficient and practical method of antigen delivery and as induction of a protective immune response against various intracellular pathogens [13-15]. DNA vaccines have been used in experiments against human immunodeficiency virus, tuberculosis, Chagas disease, and leishmaniasis [1623]. These plasmids contain cytosine-phosphate-guanosine (CpG) motifs, which bind and activate toll-like receptor 9 (TLR9), thus triggering the innate immune response and subsequent development of adaptative immunity, inducing the development of the Th1 response [24, 25]. In this context, this vaccination technology plays a promising role in the development of new candidates against CL, which requires Th1 cell immunity.

In the present work, the prophylactic potential of an intramuscular injection of plasmid DNA encoding the LmxMBA protein (membrane-bound acid phosphatase of Leishmania mexicana) was evaluated in $\mathrm{BALB} / \mathrm{c}$ mice. Immunoinformatic tools selected the $L$. mexicana membrane-bound acid phosphatase (XP_003874608.1). LmxMBA protein was identified in the L. mexicana promastigote and amastigote stages by antibodies from mice immunized with recombinant protein. Due to the high conservation found in the amino acid sequence of this protein in different Leishmania species, the extramembrane region was cloned, purified, and evaluated as a DNA vaccine candidate in $\mathrm{BALB} / \mathrm{c}$ mice against infection caused by this parasite. The vaccine efficacy was evaluated by measuring the size of the lesion in the footpad, the parasite load, and histopathological analysis of the lesion.

\section{Materials and Methods}

2.1. Cell Culture. L. mexicana promastigotes, strain MHOM/MX/92/UADY68, were axenically cultured at $28^{\circ} \mathrm{C}$ in RPMI-1640 medium (GIBCO), pH 7.4, supplemented with $10 \%$ fetal bovine serum and antibiotics $(100 \mathrm{IU} / \mathrm{ml}$ penicillin and $50 \mu \mathrm{g} / \mathrm{ml}$ streptomycin).

2.2. Animals. Female BALB/c mice (4-6 weeks old) were provided by the Animal Production and Experimentation Unit (UPEAL-CINVESTAV) following the specifications of the Mexican National Norm (NOM-062-ZOO-1999) that is a version of the Guide for the Care and Use of Laboratory Animals 2011. The research protocol (no. 0216-16) was approved by CINVESTAV's Institutional Animal Care and Use Committee (CINVESTAV-IACUC).

2.3. In Silico Analysis. L. mexicana annotated proteins from the TriTryp databases consisted of 8250 open reading frames (ORFs) (TriTrypDB-6.0_LmexicanaMHOMGT2001U1103_ AnnotatedProteins). The ORFs were analyzed for the identification of signal peptides and transmembrane helices by TMHMM (http://www.cbs.dtu.dk/services/TMHMM/1) and TOPCONS (http://topcons.cbr.su.se/). The expression sequence tags (EST) and mass spectrometry (MS) data from the TriTryp site (https://tritrypdb.org/tritrypdb/) were examined to define the expressed transmembrane proteins. Consensus subcellular localization was determined by Euk-
mPloc (http://www.csbio.sjtu.edu.cn/bioinf/euk-multi-2/), LocTree3 (https://rostlab.org/services/loctree3/), DeepLoc (http://www.cbs.dtu.dk/services/DeepLoc/), and CELLO (http://cello.life.nctu.edu.tw/). Fold change in expressed genes between promastigotes, axenic amastigotes, and macrophage-derived amastigotes was identified based on RNA Seq Evidence (Transcriptomic in https://tritrypdb.org/ tritrypdb/).

2.4. Construction of Recombinant Plasmids. The eukaryotic expression vector pVAX1 (Invitrogen, Carlsbad, CA, USA) and the bacterial expression vector pCR4-TOPO (Invitrogen, Carlsbad, CA, USA) were chosen to clone and express the LmxMBA gene. Briefly, the 1337 bp DNA fragment containing the LmxMBA gene (GenBank No. XM_003874559.1, sequence positions 94-1431), lacking the transmembrane regions, was obtained by polymerase chain reaction (PCR) (Thermal Cycler spp., Thermo Fisher Scientific) using the genomic DNA of L. mexicana promastigotes as the template and the primers LmxMBA F HindIII ( $5^{\prime}$-AAGCTTTCGCCA CCATGGACAAGGTGGAGCTGGTGCAG- ${ }^{\prime}$ ) and LmxMBA/R EcoRI (5'-CACGAATTCTTACCCGCCGC TGGACATGGGCGAC- $3^{\prime}$ ). The PCR reaction contained $1 \mu \mathrm{g}$ of template, $2 \mathrm{mM} \mathrm{MgCl}, 0.2 \mathrm{mM}$ of each dNTP, $1 \mathrm{x}$ PCR buffer, and 2.5 U of Taq DNA polymerase. PCR conditions involved initial denaturation at $94^{\circ} \mathrm{C}$ for $5 \mathrm{~min}, 35$ cycles of primer annealing at of $94^{\circ} \mathrm{C}$ for $1 \mathrm{~min}, 65^{\circ} \mathrm{C}$ for $30 \mathrm{~s}$, and $72^{\circ} \mathrm{C}$ for $1 \mathrm{~min}$ and a final extension at $68^{\circ} \mathrm{C}$ for $7 \mathrm{~min}$. The amplified fragment was purified (QIAEX II, Invitrogen, Carlsbad, CA USA), cloned into pCR4-TOPO vector and subcloned into pVAX1. The recombinant plasmid pVAX1::LmxMBA was confirmed by double restriction enzyme digestion (HindIII/EcoRI) and DNA sequencing.

The prokaryotic expression vector pRSET A (Invitrogen, Carlsbad, CA, USA) was chosen to clone and express the recombinant protein LmxMBA. The primers used were LmxMBAP/F BamHI (5'-CGGATCCTACAAGGTGGA GCTGGTGCAGGTG-3') and LmxMBAP/R HindIII (5' -GAAATATAAGCTTACCCGCCGCTGGACATGGGCG

AC- $\left.3^{\prime}\right)$. The content reaction and conditions of $\mathrm{PCR}$ reaction were previously described [26]. The amplified fragment was purified and inserted into the BamHI and HindIII restriction sites of pRSET A, obtaining the recombinant plasmid pRSETA::LmxMBA. The construct was sequenced to confirm the correct sequence of the gene after PCR and correct insertion of the gene in frame with the ATG of the plasmid.

2.5. DNA Purification. Plasmid pVAX1::LmxMBA was maintained and propagated in E. coli DH5. Endotoxin-free plasmid DNA was purified by anion-exchange chromatography using a PureLink ${ }^{\mathrm{TM}}$ HiPure Plasmid DNA Purification Kit (Invitrogen) according to instructions from the manufacturer, and DNA used for immunizations was resuspended in PBS pH 7.4. After purification, plasmid concentration was determined by absorbance at $260 \mathrm{~nm}$ and $280 \mathrm{~nm}$. The OD 260/280 ratios for purified DNA were 1.8-2.0, indicating that preparations were free of major protein contamination. 
2.6. Purification Recombinant Protein. To obtain the recombinant protein LmxMBA, E. coli BL21 pLysS cells were transformed with the recombinant plasmid pRSETA::LmxMBA and grown in Luria Bertani medium to an optical density of 0.6 at $540 \mathrm{~nm}$. Cells were induced by incubation with $0.1 \mathrm{mM}$ IPTG at $37^{\circ} \mathrm{C} / 2 \mathrm{~h}$. The cells were then harvested by centrifugation, washed in ice-cold $50 \mathrm{mM}$ Tris HCl-buffer (pH 7.5), and suspended in extraction buffer $(50 \mathrm{mM}$ Tris HCl-buffer (pH 7.5), $150 \mathrm{mM} \mathrm{NaCl}, 10 \mathrm{mM} \mathrm{MgCl}_{2}, 5 \mathrm{mM}$ $\mathrm{B}$-mercaptoethanol, $3 \mathrm{M}$ guanidinium chloride, and $2 \mathrm{M}$ urea). After disruption by sonication, the crude extract was clarified by centrifugation at $30,000 \times \mathrm{g}$ for $30 \mathrm{~min}$. The rLmxMBA was expressed as a fusion protein with an $\mathrm{N}$ terminus six-histidine-residue affinity tag and was purified, under denatured conditions by affinity chromatography using Ni-agarose resin (Qiagen, Hilden, DE) according to the manufacturer instructions. The collected protein was dialyzed for $48 \mathrm{~h}$ at $4^{\circ} \mathrm{C}$ against PBS.

2.7. Anti-rLmxMBA Antibodies. The recombinant protein LmxMBA (rLmxMBA) was obtained as described above, and female $\mathrm{BALB} / \mathrm{c}$ mice were immunized with $10 \mu \mathrm{g} / \mathrm{dose}$. Animals received only two intraperitoneal (i.p.) doses with 15 days of interval; the immunization doses were administrated in TiterMax adjuvant (1:1 mixture) (Sigma, St. Louis, MO, USA), and 30 days after the immunization scheme, mice were bled by cardiac puncture to obtain immune sera.

2.7.1. Expression of LmxMBA Antigen In Vitro. Mammalian cells were transfected with pVAX1::LmxMBA, using the XtremeGENE HP Transfection Reagent (Roche Diagnostics, Penzberg, DE), according to the manufacturer's instructions. The HeLa transfected cells were harvested $72 \mathrm{~h}$ later and washed three times with PBS. Total RNA was extracted with the TRIzol reagent (Invitrogen, Carlsbad, CA, USA). Complementary DNA (cDNA) was derived from RNA with the SuperScript ${ }^{\mathrm{TM}}$ First-Strand Synthesis System (Invitrogen, Carlsbad, CA, USA). LmxMBA cDNA was amplified by PCR using specific primers LmxMBA/F and LmxMBA/R, as described above. The amplified products were analyzed on a $1 \%$ agarose gel. In addition, the expression of LmxMBA was evaluated by immunoblotting of transfected cells. Glyceraldehyde-3-phosphate dehydrogenase (GAPDH) was used as a housekeeping control gene. The primers used were GAPDH/F ( $5^{\prime}$-GGTGCTAAGCAGTTGGTGGT- $\left.3^{\prime}\right)$ and GAPDH/R ( $5^{\prime}$-GAGTCAACGGATTTGGTCGT-3').

2.8. SDS-PAGE and Immunoblotting. Proteins were resolved on $12 \%$ SDS-PAGE and either visualized by Coomassie blue or electrophoretically transferred onto a nitrocellulose membrane for immunoblotting. Anti-histidine antibodies at $1: 1000$ dilution and anti-rLmxMBA antibodies at $1: 200$ dilution were used as primary antibodies in TBST $(150 \mathrm{mM}$ $\mathrm{NaCl}, 0.5 \%$ Tween $20,2 \%$ skim milk, and $10 \mathrm{mM}$ Tris $\mathrm{HCl}$ $\mathrm{pH}$ 7.4). Bound antibodies were detected using alkaline phosphatase-conjugated goat anti-mouse IgG (Zymed Labs, San Francisco, CA) diluted at 1:5000, and the blot was developed with NBT and BCIP (Invitrogen, Carlsbad, CA, USA).
2.9. Immunofluorescence Microscopy. L. mexicana promastigotes and amastigotes were washed 3 times in PBS and fixated in $4 \%$ paraformaldehyde (PFA) in filtered PBS pH 7.4 for $15 \mathrm{~min}$ at room temperature (RT). Following an additional PBS wash, the cells were resuspended in filtered PBS and then left to sediment and adhere to the surfaces glass slides for $30 \mathrm{~min}$ at RT. The fixed cells were permeabilized with cold methanol for $5 \mathrm{~min}$ and then labeled with mouse polyclonal anti-LmxMBA diluted 1:100 in PBS. A secondary FITC-conjugated goat anti-mouse IgG antibody was used for fluorescent detection of the LmxMBA protein. Slides were mounted with Vectashield and DAPI $\left(4^{\prime}, 6^{\prime}\right.$-diamino-2phenylindole).

2.10. Mouse Immunization and Experimental Infection. Fourto six-week-old female BALB/c mice (30 in total) were immunized intramuscularly. Plasmids were prepared as described and then dissolved in sterile PBS (pH 7.4) as DNA vaccines for immunization. Mice were divided randomly into three different groups of ten animals per group: one group was vaccinated with $100 \mu \mathrm{g}$ of pVAX1::LmxMBA plasmid/per mice, and as controls, one group was vaccinated with the empty plasmid, pVAX1, and another was not immunized (PBS). A three-dose immunization scheme at 15-day intervals followed by parasite challenge was adopted.

Two weeks after the last immunization, the mice of each group were challenged at the right footpad subcutaneously. Cultured promastigotes were collected, centrifuged at 350 $\mathrm{g} / 5 \mathrm{~min}$, washed three times with PBS, and counted in a Neubauer chamber. The inoculum was prepared in a total volume of $25 \mu \mathrm{l}$ of PBS containing $5 \times 10^{6}$ promastigotes. The lesion size was determined weekly by measuring the thickness of the infected and healthy footpads, using a Vernier caliper, and calculating the size difference between the paw pads. The animals were sacrificed 105 days after infection, and the hind legs were amputated at the level of the subtalar joint to obtain skin tissue from the footpad. Mice were bled by cardiac puncture, and the spleens were collected for immunological purposes.

2.11. Determination of the Weight of Lesions and Parasite Burden. The parasite burden was quantified in skin tissue by limiting dilution culture [27]. Briefly, hind paws (infected and healthy) were removed aseptically and submerged in 3\% ionized alcohol for up to 3 minutes to allow decontamination. The paws were weighed individually, and the difference between the infected and healthy paws of each mouse was taken as the net weight of the lesion. Skin sections of the footpad were obtained (approximately $20 \mathrm{mg}$ ), macerated in Eppendorf tubes with $1 \mathrm{ml}$ of RPMI 1640 medium culture (medium containing 10\% heat-inactivated SFB, $100 \mathrm{IU} / \mathrm{ml}$ penicillin, and $0.1 \mathrm{mg} / \mathrm{ml}$ streptomycin), and diluted with the same medium at a final concentration of $1 \mathrm{mg} / \mathrm{ml}$. The material was tenfold serially diluted in RPMI 1640 medium supplemented with 10\% FBS and cultured into 96-well flatbottom plates, in duplicate. The plates were incubated at $26^{\circ} \mathrm{C}$ and observed under an inverted microscope (Nikon Diaphot, Nikon, Japan) every 3 days, up to a maximum of 20 days, to record the dilutions containing promastigotes. 
The presence of mobile promastigotes was recorded at day 20 in each well, and the concentration of parasites per milligram of tissue was calculated as the reciprocal of the highest dilution that was positive for parasites. The total parasite load of the lesion was calculated with reference to the weight of the lesion. Each positive result is expressed as the log10 number of promastigotes on the graph.

2.12. Cell Proliferation Assay. Mouse splenocytes were prepared as follows [28]. Spleens were taken from immunized/challenge mice and control animals under sterile conditions, dissected, and resuspended in sterile, cold PBS (pH 7.4) containing FBS 2\%. RBCs were disrupted with lysis buffer, and the single-cell suspensions were adjusted to $2 \times 10^{6}$ cells $/ \mathrm{ml}$ with RPMI 1640 (HyClone, Logan, UT) containing 10\% FBS. The diluted cell suspensions $(100 \mu \mathrm{l} /$ well $)$ were dispensed into 96-well flat-bottom culture plates (Thermo Fisher Scientific, Rochester, NY) and restimulated with $10 \mu \mathrm{g} / \mathrm{ml}$ of rLmxMBA protein. Concanavalin A $(2 \mu \mathrm{g} / \mathrm{ml})$, unstimulated wells, and complete culture medium were used as positive, negative, and blank controls, respectively. Each splenocyte sample was plated in triplicate. The proliferative response was measured by WST-1 (Roche Diagnostics, Mannheim, DE) according to fabricant instruction. After $72 \mathrm{~h}$ of culture, $10 \mu \mathrm{l}$ of WST-1 was added to each well and incubation continued for $4 \mathrm{~h}$ at $37^{\circ} \mathrm{C}$ and $5 \% \mathrm{CO}_{2}$. Following incubation, plates were read at $450 \mathrm{~nm}$. The stimulation index (SI) was calculated as the ratio of average OD value of wells containing antigen-stimulated cells to average OD of wells containing only cells with the medium.

2.13. Histological Analysis. Slices taken from the skin of the footpad were rinsed with PBS and fixed with $10 \%$ formaldehyde for $24 \mathrm{~h}$. Fixed sections were embedded in paraffin, sectioned $(6 \mu \mathrm{m})$, and stained with hematoxylin-eosin. The presence of amastigotes and histopathological alterations were examined under a light microscope (Nikon Eclipse $80 \mathrm{i}$, Nikon, Japan). Semiquantification of parasites per field was expressed in ranges of parasites observed in 10 consecutive fields of the microscope under the 100x objective. At least 100 microscopic fields were examined before a sample was reported as negative.

2.14. Isotyping of Antibodies. The IgG1, IgG2a, IgG2b, IgG3, $\operatorname{IgA}$, and IgM immunoglobulins from DNA-vaccinated mice were evaluated by indirect ELISA assays. Blood samples were collected ( $n=10$ animals/group) from the immunized mice 105 days after the infection. The sample was centrifuged for serum preparation. The 96-well microplates (Thermo Fisher Scientific, Rochester, NY) were coated with rLmxMBA $(2 \mu \mathrm{g} / \mathrm{ml})$ in $100 \mathrm{mM}$ carbonate-bicarbonate buffer (pH 9.6) and incubated overnight at $4^{\circ} \mathrm{C}$. Plates were washed with PBS-0.05\% Tween 20 (PBST) and incubated for $2 \mathrm{~h}$ at $37^{\circ} \mathrm{C}$ with blocking solution (PBS containing 5\% skim milk). After washing with PBST, diluted serum samples $(1: 100)$ in PBST were added to each well and incubated at $37^{\circ} \mathrm{C}$ for $1 \mathrm{~h}$ and washed 3 times after the reaction. As a negative control, a pool of preimmune sera was used in all experiments. For isotyping, the plates were incubated with peroxidase-labeled goat anti-mouse isotype antibodies (Abcam, Cambridge, UK) at $1: 10000$ dilution in PBS-T for $2 \mathrm{~h}$ at RT and then washed 4 times with PBST and PBS. The peroxidase activity was developed by 3-ethylbenzthiazoline-6-sulfonic acid (ABTS) substrate, and the optical density (OD) was read at $405 \mathrm{~nm}$ in an ELISA microplate reader (LabSystem Multiskan MS).

2.15. Statistical Analysis. The results were expressed as means \pm standard errors of the mean (SEM). All data in the study were compared using the Statistical Package for the Social Sciences (SPSS) 23.0 Data Editor (SPSS Inc., Chicago, Illinois, USA). Statistical analysis was performed using one-way analysis of variance (ANOVA) followed by a Tukey post hoc test to identify significantly different groups. Differences were considered statistically significant when the $p$ value was $<0.05$.

\section{Results}

3.1. Bioinformatic Analysis. We carried out a bioinformatic analysis of the L. mexicana ORF database to identify new proteins as potential candidates for the development of a vaccine. The main criterion was to select plasma membrane proteins. Analysis of the 8250 ORFs by using TMHMM [29] and TOPCONS [30] showed that 1543 proteins had transmembrane helices, and of the latter, 186 had the signal peptide. These membrane proteins were type I (112 proteins) and type III (74 proteins). Examination of the EST and MS/TriTrypDB databases indicated that 50 type I transmembrane proteins were expressed, and they presented the following subcellular location: 13 excreted, 7 in mitochondria, 8 in plasma membrane, 3 excreted/plasma membrane, 7 in endoplasmic reticulum, and 12 in various locations [31-34]. The transcriptomic analysis of the plasma membrane and plasma membrane-excreted proteins showed that the membranebound acid phosphatase is expressed at the same level in axenic amastigotes, macrophage-derived amastigotes, and promastigotes as compared with other proteins (Figure 1).

3.2. LmxMBA Localization in Leishmania mexicana. The subcellular location of LmxMBA was investigated in promastigotes and amastigotes by indirect immunofluorescence. Permeabilized and nonpermeabilized cells were incubated with the polyclonal anti-LmxMBA antibody, followed by a secondary antibody conjugated to FITC (green). As shown in Figure 2, green fluorescence was associated with the base of the flagellum in both permeabilized and nonpermeabilized promastigotes. Permeabilized promastigotes also showed green fluorescence between the nucleus and the kinetoplast. In amastigotes, green fluorescence was associated mainly towards one end of the parasite membrane.

3.3. LmxMBA Expression in Mammalian Cells. The recombinant plasmid pVAX1::LmxMBA was constructed to investigate the potential of the LmxMBA gene as a prophylactic DNA vaccine, in response to cutaneous leishmaniasis in the murine model. To ensure that LmxMBA could indeed be transcribed in mammalian cells, the presence of specific mRNA was established by RT-PCR from total RNA isolated 


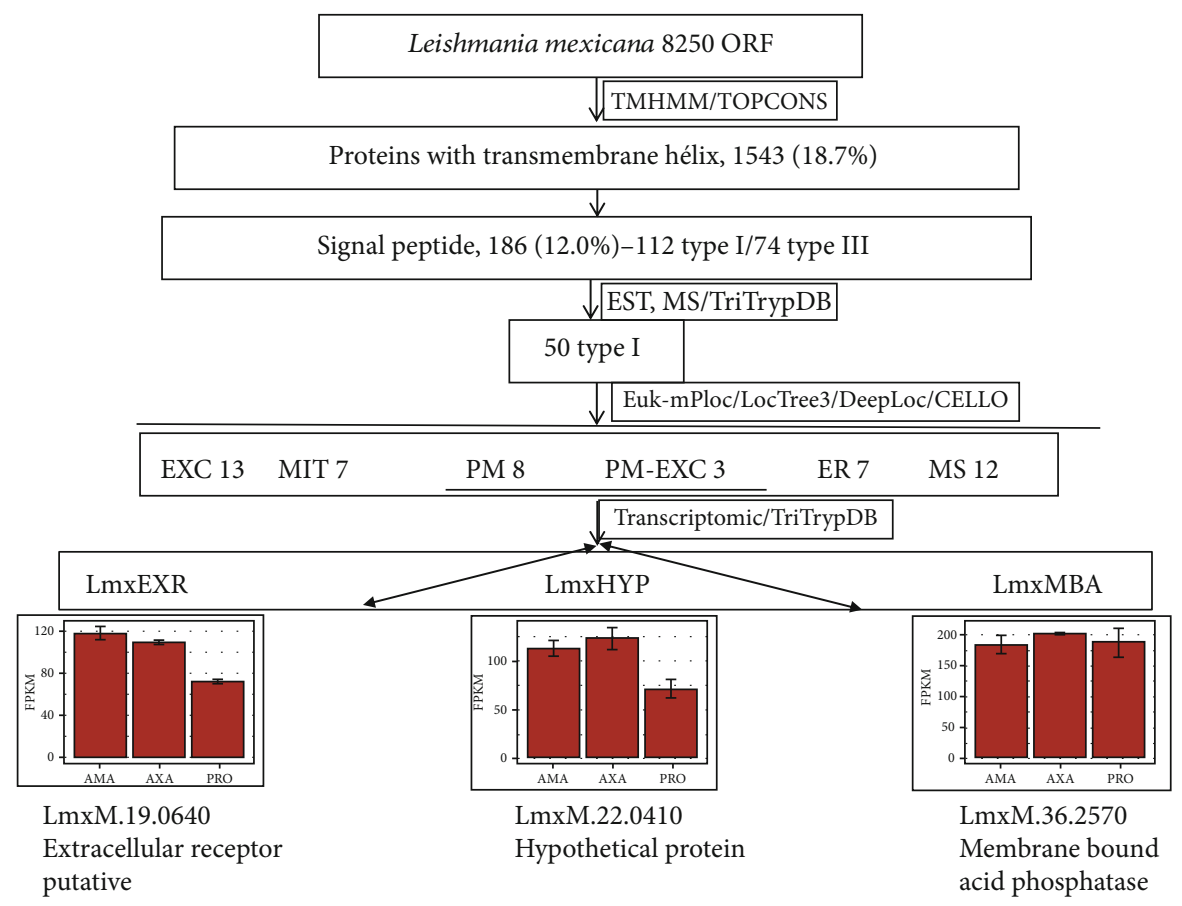

FIgURE 1: Strategy to identify putative vaccine candidates in L. mexicana. Abbreviations: EXC: excreted; MIT: mitochondria; PM: plasma membrane; ER: endoplasmic reticulum; MS: multiple sites.

from HeLa cells transfected with plasmid encoding the LmxMBA protein. RT-PCR showed amplification products at $400 \mathrm{bp}$ for GAPDH in all the groups of cells (Figure 3(a)), while only the pVAX1::LmxMBA transfected cells showed amplification of the LmxMBA mRNA at $1337 \mathrm{bp}$, not so in untransfected cells or transfected with the empty vector (Figure 3(a)). In addition, the LmxMBA protein was detected in lysates of HeLa cells transfected with the plasmid encoding that protein by western blotting analysis (Figure 3(b)). The expected MW for LmxMBA protein is $49 \mathrm{kDa}$, and possibly, the lower and higher bands are degradation product and protein aggregate of the same protein, respectively.

3.4. Effect of DNA Vaccination on Lesion Development. Weekly measurements followed lesion development, and the lesion size kinetics showed that infection caused a progressive increase in footpad swelling in all mice from control groups. Mice immunized with plasmid encoding LmxMBA gene showed a delayed increase in lesion diameter compared to control mice, immunized with PBS $(p<0.0001)$ or pVAX1 $(p<0.05)$. In addition, statistical significance in the size of the lesion was found from 60 to 105 days after infection compared to the group immunized with PBS $(p<0.0001)$ and with pVAX1 $(p<0.05)$. Vaccination of mice with the empty plasmid pVAX1 showed continuous growth of the lesion at a slower rate than that observed in mice immunized with PBS $(p<0.05)$ (Figure 4(a)).

3.5. Determination of Parasite Load. There was a good correlation between lesion sizes and parasite loads. A significantly lesser amount of parasites was observed in footpad lesions of mice vaccinated with pVAX1::LmxMBA plasmid, compared to the control groups inoculated with the empty vector $(p<0.001)$ or PBS $(p<0.0001)$. The footpad of mice immunized with the recombinant plasmid also showed lower lesion weights at 105 days compared to control mice immunized with PBS $(p<0.01)$ and compared to the group immunized with pVAX1. No statistically significant differences were found $(p>0.05)$ (Figures $4(\mathrm{~b})$ and $4(\mathrm{c}))$.

3.6. Histopathological Study. Histopathological study showed a lesser amount of parasites in the tissue skin of mice immunized with the plasmid pVAX1::LmxMBA (Figure 5). 80\% of the animals were negative in the microscopic examination of amastigotes, and the remaining $20 \%$ showed few parasites (020 per field) in the dermis. Mice immunized with PBS or with the empty plasmid, pVAX1, showed a high number of amastigotes (>200 per field) at the site of inoculation (Figures 5(a) and 5(b)). Besides, an infiltrate of inflammatory cells occupying the dermis and hypodermis with the presence of multiparasitic vacuolated macrophages was observed. A disorganized dermal region was also observed, with loss of normal morphology, filled by an intense infiltrate, rich in polymorphonuclear and mononuclear cells (Figures 5(a) and 5(b)). Mice immunized with the empty plasmid pVAX1 showed fewer vacuolated macrophages compared to nonimmunized mice. However, highly parasitized nonvacuolated macrophages were observed in mice immunized with the empty vector.

In contrast, immunized mice with pVAX1::LmxMBA plasmid showed a lower amount of parasitic structures per macrophage at the site of the lesion. The lesion of these animals showed a lower frequency of parasitized macrophages 

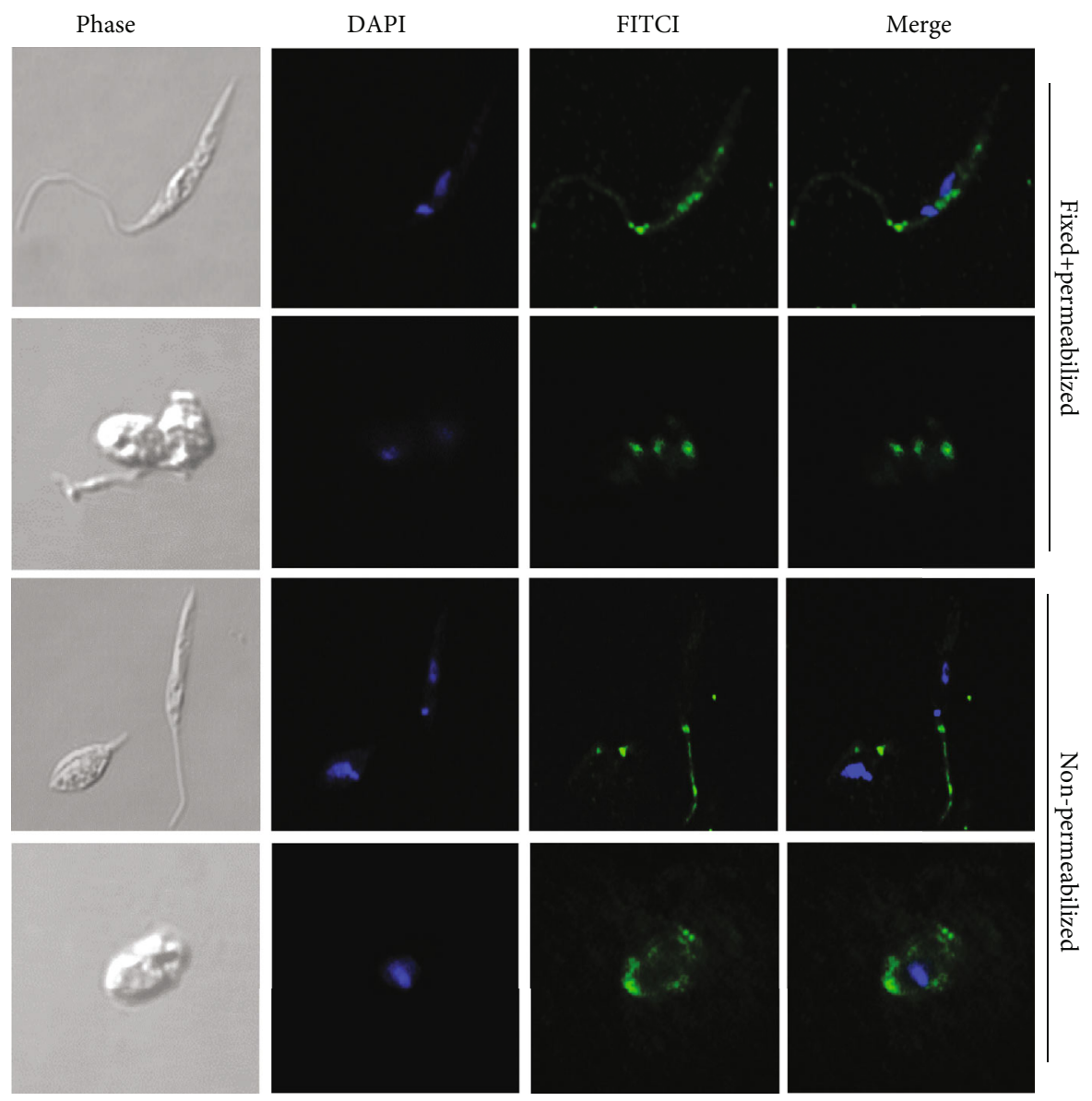

Figure 2: Subcellular localization of LmxMBA protein in L. mexicana promastigotes and amastigotes. Immunofluorescence microscopy utilizing serum anti-LmxMBA and secondary antibody coupled to FITC; nucleus and kinetoplast were marked with DAPI.

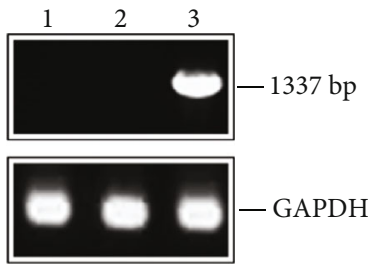

(a)

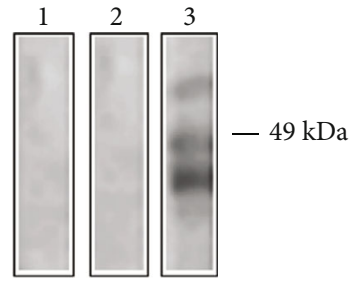

(b)

FIgure 3: Expression of LmxMBA in transfected human HeLa cells. Nontransfected HeLa cells (1) and transfected with pVAX1 (2) or with pVAX1::LmxMBA (3) were assayed by RT-PCR (a) and immunoblotting (b) for the expression of LmxMBA $48 \mathrm{~h}$ posttransfection.

(20\%) or total absence of them (80\%) (Figure 5(c)). Vaccination with pVAX1::LmxMBA plasmid showed the typical histological characteristic in the infected footpad; a dermis with abundant collagen fibers was observed by microscopic examination, similar to control tissue (Figure 5(d)). Although there was still a mild inflammatory infiltrate in the dermis of some animals (30\%) (including those with a low number of parasites per field), no damage or loss of the tissue microarchitecture was observed.

3.7. Lymphocyte Proliferation Assay. To further investigate the cellular immune response elicited by immunization with
pVAX1::LmxMBA, cellular proliferation assays were performed. As shown in Figure 6, mice immunized with pVAX1::LmxMBA induced a greater antigen-specific cell proliferation response, in contrast to the control groups $(p<0.05)$. This showed the ability of LmxMBA to induce spleen cell proliferation as a maker of memory $\mathrm{T}$ cell immune responses whereas no significant difference was observed between pVAX1 and PBS groups $(p>0.05)$.

3.8. Isotyping of Antibodies. In order to investigate the phenotype (Th1/Th2 pattern) of immune response elicited by immunization of pVAX1::LmxMBA, the isotype of 


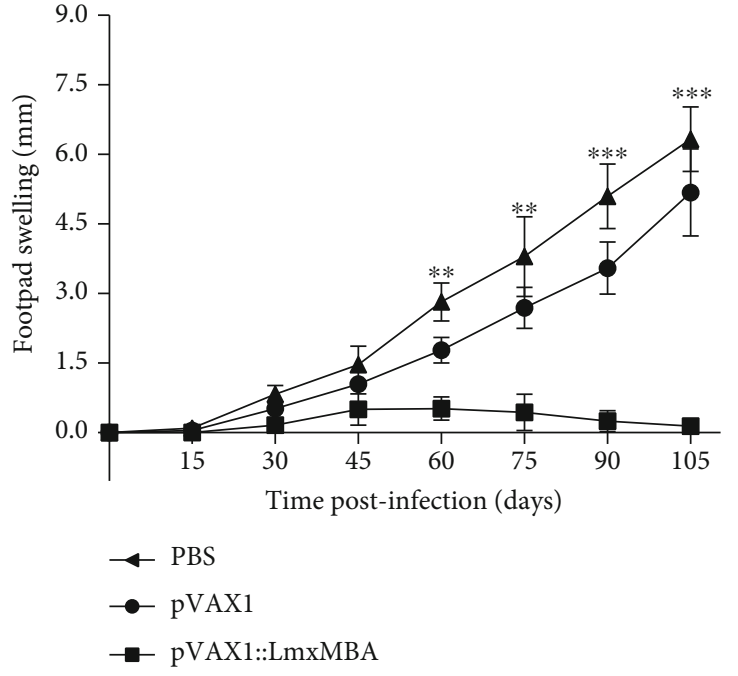

(a)

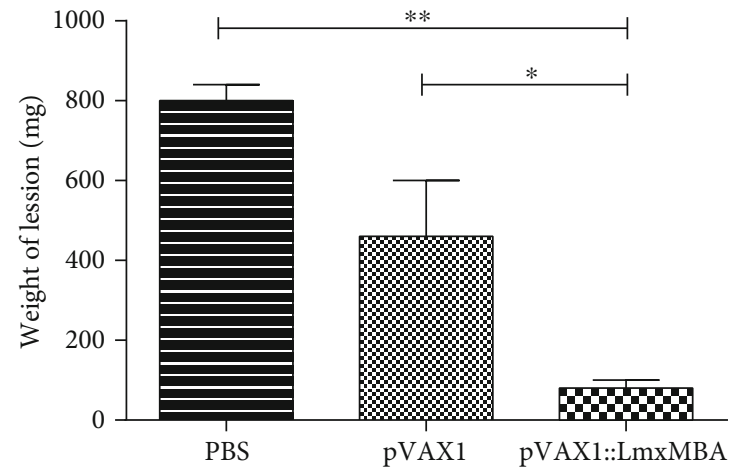

(b)

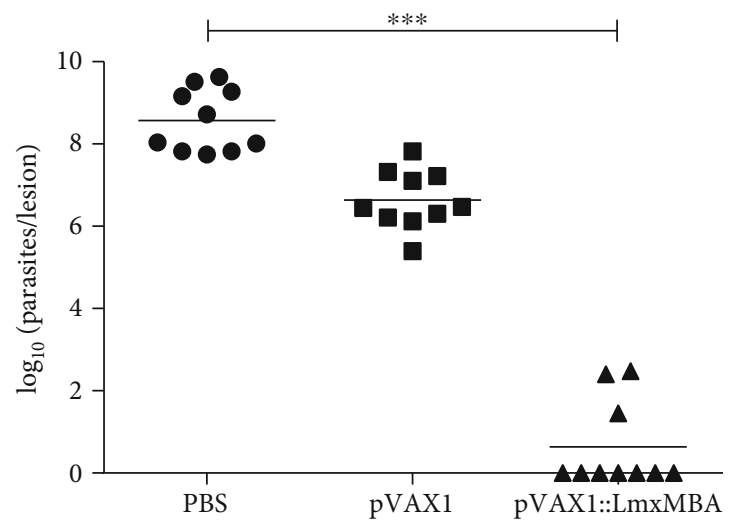

(c)

FIGURE 4: Footpad swelling (a), weight of lesions (b), and parasite burden (c) in BALB/c mice immunized and challenged with promastigotes of $L$. mexicana. Mice were immunized three times (intervals of 15 days between doses) with pVAX1::LmxMBA, pVAX1, or PBS. The results are expressed as means \pm standard errors of the mean (SEM) of ten mice per group (a). The weight of the lesion was determined by amputation of the paw 105 days after the infection; the difference between the infected and healthy paws was taken as the net weight of the lesion (b). The number of parasites in the lesion was determined by limiting dilution culture, and the results are expressed as $\log _{10}$ (c). ${ }^{*} p<0.05$.

antibodies was analyzed by an indirect ELISA method. As shown in Figure 7, anti-LmxMBA IgG+M+A values were significantly lower than the PBS control group $(p<0.01)$, and there was no statistically significant difference with the pVAX1 control group $(p>0.05)$. When comparing the immunoglobulin isotype levels from different groups of mice, we observed a low level of IgG1 in animals immunized with pVAX1::LmxMBA and the highest level in the control group immunized with PBS $(p<0.001)$ and pVAX1 $(p<0.05)$. On the other hand, a statistically significant difference in IgG2a levels was detected between mice immunized with pVAX1::LmxMBA and control mice, pVAX1 $(p<0.001)$ or PBS $(p<0.001)$. In all groups of mice, the levels of IgG3 and IgA remained low. The IgG2a/IgG1 ratio was $>1$ and higher in immunized mice with pVAX1::LmxMBA compared to control mice, PBS $(p<0.05)$ or pVAX1 $(p<0.05)$. This ratio does not differ significantly between mice immunized with PBS and pVAX1 $(p>0.05)$ (Figure 7).

\section{Discussion}

The control of leishmaniasis through vaccination is an increasing challenge. Although several attenuated and protein-based vaccines have protected against different species of Leishmania in different animal models, some of them have intrinsic disadvantages, such as the risk of reversion to virulence and the selection and use of adjuvants, respectively $[8,10,34]$. Vaccination with plasmid DNA is an active area of research applied to cancer and microbial pathogens associated with intracellular infections $[19,35]$. DNA vaccines have been reported to persist in skeletal muscle for at least 19 months and are easy to produce in high purity $[36,37]$. DNA vaccines are temperature stable (room temperature 2 to $8^{\circ} \mathrm{C}$ ) over extended periods, enabling easier shipping and storage and allows to dispense with the cold chain used in conventional vaccines $[37,38]$. Several plasmids used as DNA vaccines are stable for more than 3 years at $4^{\circ} \mathrm{C}$, at least 


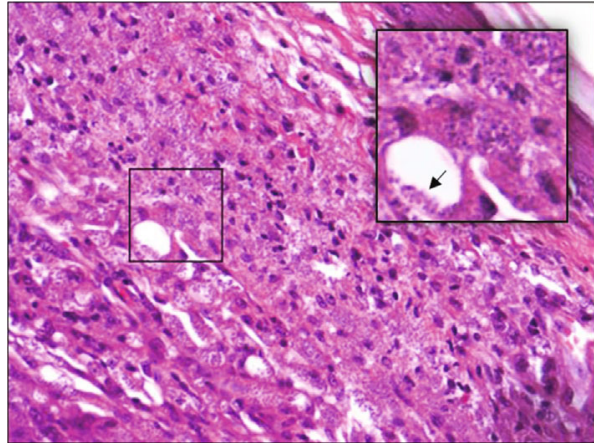

(a)

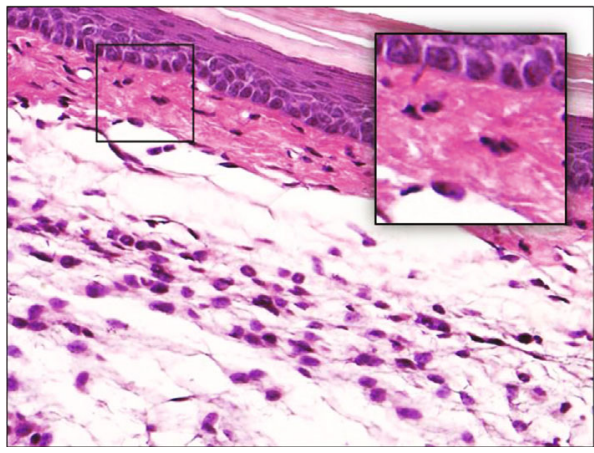

(c)

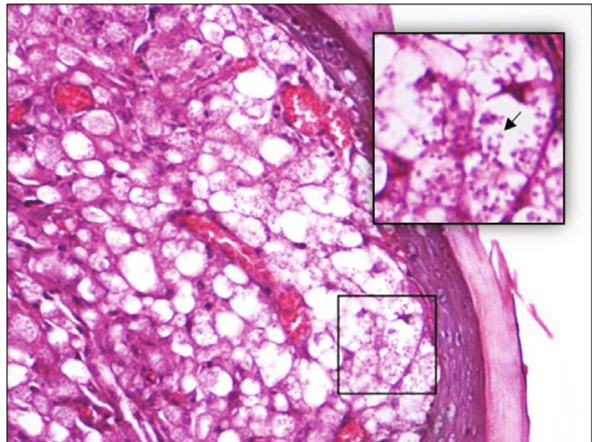

(b)

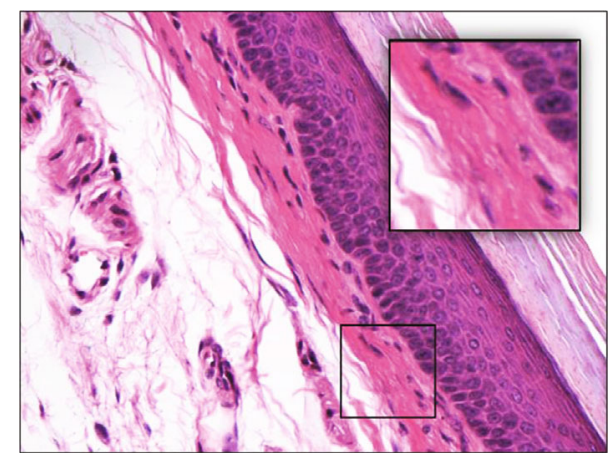

(d)

FIGURE 5: Histopathological analysis of the footpad of mice immunized with pVAX1::LmxMBA and challenged with promastigotes of $L$. mexicana. Tissues were collected 105 days after infection, and the sections were stained with hematoxylin and eosin. Vacuolated macrophages containing amastigotes were abundant in footpad lesions of mice immunized with PBS (b), as compared with mice immunized with the empty vector, pVAX1 (a); the dermis of control animals showed a massive infiltration with parasitized macrophages (arrows). In contrast, mice immunized with pVAX1::LmxMBA displayed more preserved skin with lymphocytes and macrophages containing few or no parasites (c). Microarchitecture of the dermis in mice immunized with pVAX1::LmxMBA was globally preserved in a similar manner to the healthy footpad (d).

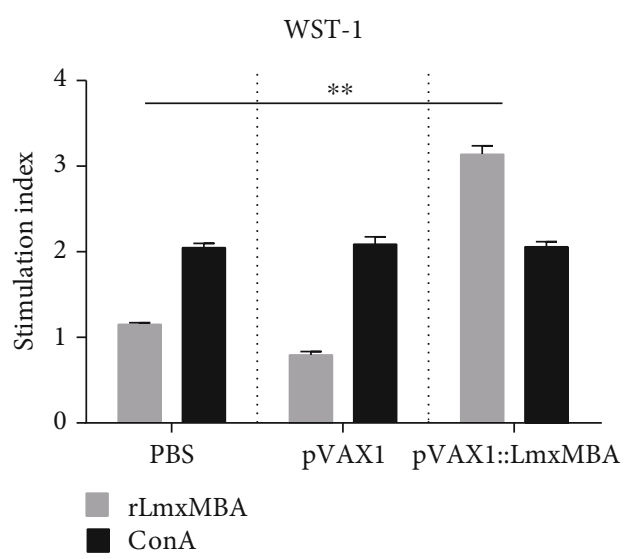

FIgURE 6: Proliferation of mononuclear cells in the spleen of mice immunized with PBS, pVAX1, or pVAX1::LmxMBA after stimulation with rLmxMBA. The mean stimulation index (SI) of vaccinated mice (injected with pVAX1::LmxMBA) was nearly 3 times greater than the mean SI of control mice (injected with PBS or pVAX1). ConA: concanavalin A. ${ }^{*} p<0.05$.

1 year at $25^{\circ} \mathrm{C}$, and 1 month at $37^{\circ} \mathrm{C}$ [39]. DNA vaccination has been used against different diseases due to its potential to induce both humoral and cellular immune responses and together with reverse vaccinology represents a potentially new approach to design vaccines against leishmaniasis and other infectious diseases such as hepatitis B and C, malaria, and human papillomavirus infection $[38,39]$.

We identified a potential candidate for a protective vaccine by reverse vaccinology. The criteria by which we chose to select potential vaccine antigen initially included a focus on surface proteins and sequence conservation among Leishmania species. Other considerations were that the chosen candidate must lack sequence identity with human proteins and the ability to express the protein in a high-throughput manner for vaccine production, e.g., we discard insoluble proteins with more than two transmembrane helices. In this regard, the LmxMBA protein has a $26.9 \%$ identity with a human protein. However, importantly, it has no shared stretches greater than six amino acids and is therefore unlikely to contain any MHC I or II epitopes. Therefore, this protein is expected to have no safety concerns if administered during vaccine trials.

Infection with L. mexicana causes many CL cases in the Americas and, less frequently, diffuse cutaneous and mucocutaneous leishmaniasis, even more disfiguring forms of the disease. L. major can also cause CL in the old world, and on the other hand, the deadliest form of the disease, VL, is mainly caused by $L$. donovani and L. infantum [40-42]. 

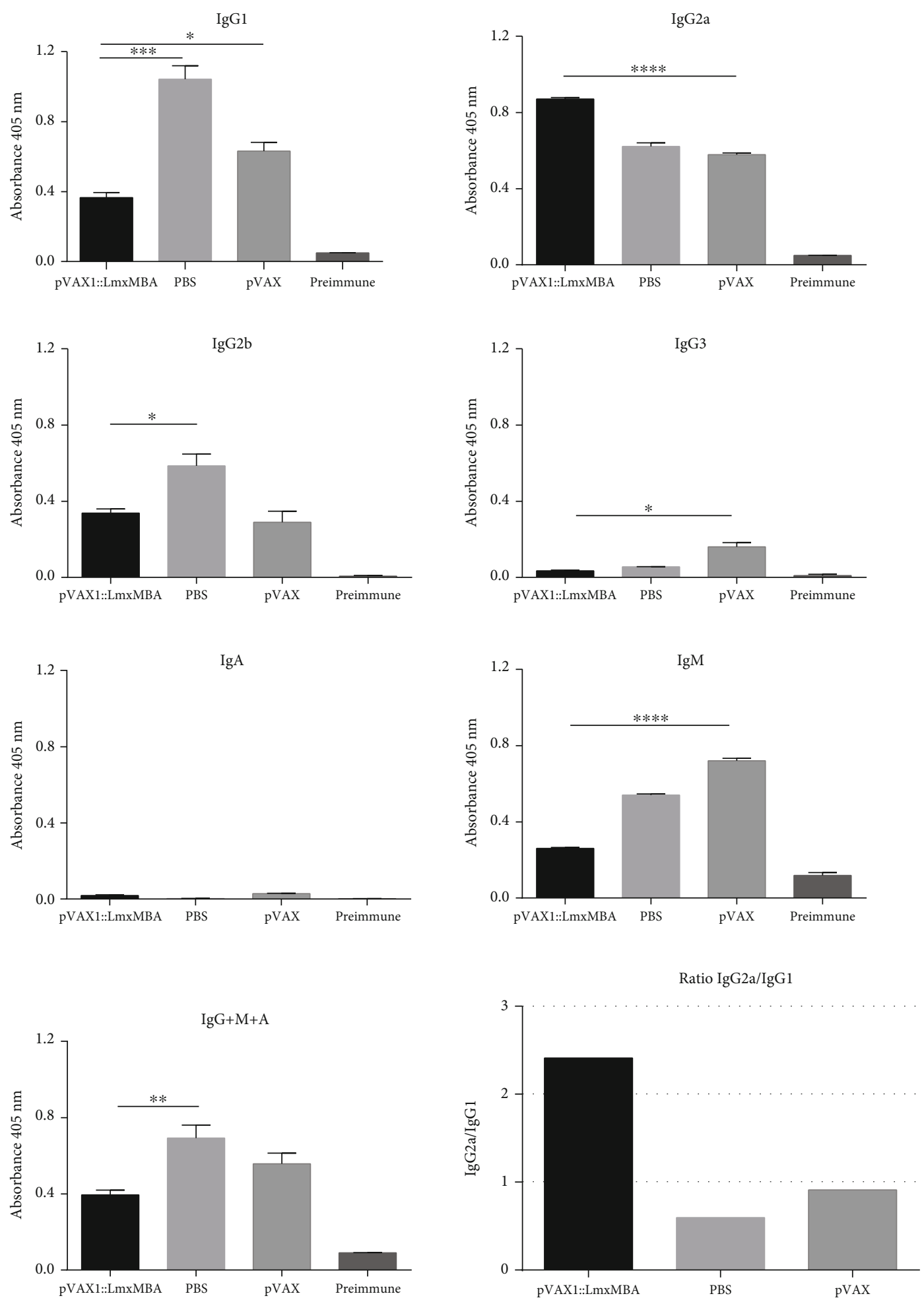

FIGURE 7: Vaccine-specific serological responses. Serum samples were collected by cardiac puncture on day 105 post infection. Plates were coated with $2 \mu \mathrm{g} / \mathrm{ml}$ of rLmxMBA, and serum samples were diluted 1:100. Each bar represents the average \pm standard deviation of optical densities. ${ }^{*} p<0.05$.

Thus, when the selected antigen is conserved in the various Leishmania species, it is possible that a vaccine directed against CL could protect against these other forms of leishmaniasis. In this way, the candidate vaccine could confer cross-protection against other Leishmania species because the extramembrane region of the LmxMBA protein has $>90 \%$ identity with $L$. major, $L$. donovani, and $L$. infantum homologues. Even when considering the most distant species that belong to the alternated subgenus Viannia, the candidate antigen exhibits at 
least $71 \%$ sequence conservation with $L$. braziliensis, $L$. panamensis, and L. guyanensis species.

LmxMBA is a protein exposed on the membrane of $L$. mexicana and belongs to the acid phosphatase expressed in the flagellar pocket in promastigotes and amastigotes. The LmxMBA gene [43] and its homologues in Trypanosoma brucei [44] encode proteins that have been observed in small vesicle-like structures close to the flagellar pocket, and protein overexpression in L. mexicana promastigotes leads to the translocation of the protein to the cell surface and the flagellar pocket membrane.

In this study, we found the LmxMBA protein is located mainly in the flagellar pocket membrane of wild-type promastigotes and amastigotes. It is less frequent on the surface or in organelles of the parasite, including the flagellum. Proteins exposed on the plasma membrane have been shown to represent the most accessible pool of potential vaccine targets for the development of vaccines. Therefore, LmxMBA as a membrane-bound protein may represent an ideal candidate for the development of a DNA vaccine against L. mexicana.

There are several key components involved in the immune response to Leishmania infection. The outcome of infection depends on the host's ability to mount a protective Th1 response versus the ability of the parasite to evade and manipulate the host's immune system [45]. Macrophages and effects molecules, dendritic cells, T helper cells, cytotoxic $\mathrm{T}$ cells, natural killer cells, and cytokines are all considered to play essential roles in the immune response to Leishmania infection [45]. In this study, DNA vaccine encoding LmxMBA of L. mexicana was able to promote humoral and cellular immune responses, reducing the clinical manifestations of the cutaneous disease in genetically susceptible mice. Most animals (70\%) did not show any footpad swelling at 105 days after challenge, the parasites were cleared from the injection site, and the dermis was free of inflammatory inflates. The rest of the animals in this group (30\%) showed minimal and transient swelling at the inoculation site and a low number of parasites and mild inflammatory infiltrates. A delay in the development of the disease was observed in all animals immunized with the LmxMBA gene. In cutaneous infection with $L$. major, vaccination with the divergent part of $\mathrm{H} 2 \mathrm{~B}$ protein confers clinical and parasitological protection against a virulent challenge, considered a protective effect when the size of the lesion and the parasite load in the vaccinated mice were significantly smaller than that observed in control animals [46]. In the experimental mouse model of visceral leishmaniasis caused by $L$. donovani, Melby et al. [47] showed that DNA vaccination using a cDNA library, containing notably $\mathrm{H} 2 \mathrm{~B}$, partially protects $\mathrm{BALB} / \mathrm{c}$ mice against a virulent challenge with $L$. donovani. The protective effect induced by the vaccine was partial since, during infection in this experimental model, vaccination was more efficient at clearing parasites from the liver than the spleen.

Immunization with the empty plasmid generated macroscopical lesions in all of this group's animals (100\%). However, the size was smaller than the PBS control group's lesions, the parasite load remained high, and there was tissue damage with chronic inflammatory infiltrates. This apparent protective effect may be due to the CpG motif. The empty vector has CpG motifs that act as a "danger signal" and as an enhancer of the Th1 immune response in DNA vaccination through interaction with TLR9-positive cells, and this could be activating a nonspecific immune response against infection $[24,25]$, thus inducing a lower swelling without reducing the parasite load.

As expected, parasites in the lesion were evident in all the animals in the control groups by staining tissue. However, in the group of animals immunized with the candidate antigen, only $20 \%$ showed parasites by stained skin and $10 \%$ more by limiting dilution culture; this is consistent with other studies in mice since limiting dilution culture allows quantification of parasite burdens at least 50- to 100 -fold lower than the minimal parasite burdens quantifiable on stained organs [48]. Due to its high sensitivity, this method is particularly suitable for experimental situations in which parasites cannot be visualized in stained organs: e.g., after therapeutic intervention or a low parasite persistence. It would be interesting to extend the animals' monitoring time to observe the complete elimination of the parasite in the infected tissue or the persistence. Nevertheless, an essential characteristic of leishmaniasis is that residual parasites could remain in the host despite the lesion's disappearance, probably for a very long time [49].

The vaccine also showed that the control was given by a Th1-type response, since mice immunized with pVAX1::LmxMBA produced significantly higher levels of IgG2 compared to control mice, which conversely showed significantly high levels of IgG1. Many reports show that during Leishmania infection, higher amounts of the IgG1 isotype in comparison with IgG2a are associated with disease progression $[50,51]$. This $\operatorname{IgG} 2 \mathrm{a} / \mathrm{IgG} 1>1$ ratio is representative of a Th1-type immune response because IFN- $\gamma$, produced by Th1-like cells, suppresses the induction of IgG1 and increases the production of IgG2 antibodies [52]. On the other hand, levels of IgM, IgG2b, and IgG3 were significantly lower in the experimental group compared to the control mice. Humoral immune response has been evaluated in different stages of the disease in humans with leishmaniasis. Elevated levels of IgM antibodies have been found in the serum of patients with visceral leishmaniasis who are in the acute phase of the disease, IgM levels remain high in patients resistant to sodium stibogluconate (SAG), and patients who respond to SAG showed a decrease in the levels of total $\mathrm{IgG}, \operatorname{IgM}$, and $\operatorname{IgE}[53]$.

It has been mentioned that the deletion mutant for this gene is able to infect $\mathrm{BALB} / \mathrm{c}$ mice and peritoneal macrophages, thus suggesting that LmxMBA is neither involved in the infection process nor required for amastigote survival in the infected host cell [54]. It could be possible that the immune response induced by DNA vaccination with the LmxMBA gene affects the activity of LmxMBA or homologous proteins essential for the infection and survival of amastigotes within the host. The L. mexicana genome codifies for 3 membrane-bound acid phosphatases and 1 secreted histidine acid phosphatase with $53-56 \%$ homology to LmxMBA (data not shown).

In conclusion, immunization with the gene LmxMBA can induce partial protective immunity against $L$. mexicana 
infection in the murine model. Until now, the efficacy of the single-dose vaccine candidate has not been evaluated. Future studies would be of great interest to test our DNA vaccine under additional conditions, mainly if lower doses of the vaccine or even a single dose is sufficient to reduce the clinical characteristics of visceral and cutaneous leishmaniasis in other animal models.

Our data identify a vaccine antigen that could potentially be used in target vaccination campings in L. mexicanaendemic regions. Due to possible cross-reactivity against other Leishmania species, it would be essential to evaluate the efficacy of this DNA vaccine against the parasitic challenge with species that cause visceral leishmaniasis in large animals, including dogs and also in humans, in order to find universal vaccine leishmaniasis.

\section{Data Availability}

The data that support the findings of this study are available from the corresponding author upon reasonable request.

\section{Conflicts of Interest}

The authors declare that they have no known competing financial interests or personal relationships that could have appeared to influence the work reported in this paper.

\section{Authors' Contributions}

María Angélica Burgos-Reyes handled investigation and writing (original draft). Lidia Baylón Pacheco worked on methodology and investigation. Patricia Espíritu-Gordillo took care of formal analysis and visualization. Silvia Galindo-Gómez managed the investigation. Victor Tsutsumi secured the validation and resources. José Luis RosalesEncina made bioinformatics analysis, conceptualization, validation, funding acquisition, writing (review and editing), and thesis director of Ph.D. student MABR.

\section{Acknowledgments}

We would like to thank Mr. Enrique Martinez de Luna for his technical assistance and María Guadalupe Aguilar González from the Departamento de Genética y Biología Molecular, CINVESTAV, México, for the DNA sequencing. MABR was a recipient of a Ph.D. fellowship from Consejo Nacional de Ciencia y Tecnología (CONACyT), México (CVU 636713). This work was supported by a grant from CONACyT, México (Grant CB 2014-240882-M), and a grant from Fondo SEP-Cinvestav (Application number 193) to JLRE.

\section{References}

[1] J. Alvar, I. D. Vélez, C. Bern et al., "Leishmaniasis worldwide and global estimates of its incidence," PLoS One, vol. 7, no. 5, article e35671, 2012.

[2] WHO, "Neglected tropical diseases. Leishmaniasis. Global Health Observatory (GHO) Data 2019," August 2020, https:// www.who.int/gho/neglected_diseases/leishmaniasis/en/.
[3] L. Kedzierski, "Leishmaniasis vaccine: where are we today?," Journal of Global Infectious Diseases, vol. 2, no. 2, pp. 177$185,2010$.

[4] R. Reithinger, J.-C. Dujardin, H. Louzir, C. Pirmez, B. Alexander, and S. Brooker, "Cutaneous leishmaniasis," The Lancet Infectious Diseases, vol. 7, no. 9, pp. 581-596, 2007.

[5] F. Chappuis, S. Sundar, A. Hailu et al., "Visceral leishmaniasis: what are the needs for diagnosis, treatment and control?," Nature Reviews Microbiology, vol. 5, no. 11, pp. 873-882, 2007.

[6] J. Alexander and K. Bryson, "T helper (h)1/Th2 and Leishmania : paradox rather than paradigm," Immunology Letters, vol. 99, no. 1, pp. 17-23, 2005.

[7] L. P. Carvalho, S. Passos, A. Schriefer, and E. M. Carvalho, "Protective and pathologic immune responses in human tegumentary leishmaniasis," Frontiers in Immunology, vol. 3, pp. 1-9, 2012.

[8] J. Poot, K. Spreeuwenberg, S. J. Sanderson et al., "Vaccination with a preparation based on recombinant cysteine peptidases and canine IL-12 does not protect dogs from infection with Leishmania infantum," Vaccine, vol. 24, no. 14, pp. 24602468, 2006.

[9] A. P. Fernandes, M. M. S. Costa, E. A. F. Coelho et al., "Protective immunity against challenge with Leishmania (Leishmania) chagasi in beagle dogs vaccinated with recombinant A2 protein," Vaccine, vol. 26, no. 46, pp. 5888-5895, 2008.

[10] D. RIVIER, R. SHAH, P. BOVAY, and J. MAUEL, "Vaccine development against cutaneous leishmaniasis. Subcutaneous administration of radioattenuated parasites protects CBA mice against virulent Leishmania major challenge," Parasite Immunology, vol. 15, no. 2, pp. 75-84, 1993.

[11] S. S. A. A. Hasson, J. K. Z. Al-Busaidi, and T. A. Sallam, "The past, current and future trends in DNA vaccine immunisations," Asian Pacific Journal of Tropical Biomedicine, vol. 5, no. 5, pp. 344-353, 2015.

[12] S. Bhaumik, R. Basu, S. Sen, K. Naskar, and S. Roy, "KMP-11 DNA immunization significantly protects against $L$. donovani infection but requires exogenous IL-12 as an adjuvant for comparable protection against L. major," Vaccine, vol. 27, no. 9, pp. 1306-1316, 2009.

[13] J. B. Alarcon, G. W. Waine, and D. P. McManus, "DNA vaccines: technology and application as anti-parasite and antimicrobial agents," Advances in Parasitology, vol. 42, pp. 343410, 1999.

[14] A. M. Watts and R. C. Kennedy, "DNA vaccination strategies against infectious diseases," International Journal for Parasitology, vol. 29, no. 8, pp. 1149-1163, 1999.

[15] A. M. Watts, M. H. Shearer, H. I. Pass, R. K. Bright, and R. C. Kennedy, "Comparison of simian virus 40 large $\mathrm{T}$ antigen recombinant protein and DNA immunization in the induction of protective immunity from experimental pulmonary metastasis," Cancer Immunology, Immunotherapy, vol. 47, no. 6, pp. 343-351, 1999.

[16] R. N. Coler, A. Campos-Neto, P. Ovendale et al., "Vaccination with the $\mathrm{T}$ cell antigen Mtb 8.4 protects against challenge with Mycobacterium tuberculosis," The Journal of Immunology, vol. 166, no. 10, pp. 6227-6235, 2001.

[17] N. L. Letvin, "Progress in the development of an HIV-1 vaccine," Science, vol. 280, no. 5371, pp. 1875-1880, 1998.

[18] D. B. Lowrie, "DNA vaccines against tuberculosis," Current Opinion in Molecular Therapeutics, vol. 1, no. 1, pp. 30-33, 1999. 
[19] J. D. Boyer, M. Chattergoon, K. Muthumani et al., "Next generation DNA vaccines for HIV-1," Journal of Liposome Research, vol. 12, no. 1-2, pp. 137-142, 2002.

[20] B. Salgado-Jiménez, M. Arce-Fonseca, L. Baylón-Pacheco, P. Talamás-Rohana, and J. L. Rosales-Encina, "Differential immune response in mice immunized with the $\mathrm{A}, \mathrm{R}$ or $\mathrm{C}$ domain from TcSP protein of Trypanosoma cruzi or with the coding DNAs," Parasite Immunology, vol. 35, no. 1, pp. 3241, 2013.

[21] M. Arce-Fonseca, M. A. Ballinas-Verdugo, E. R. A. Zenteno et al., "Specific humoral and cellular immunity induced by Trypanosoma cruzi DNA immunization in a canine model," Veterinary Research, vol. 44, no. 1, p. 15, 2013.

[22] O. Rodríguez-Morales, S. C. Carrillo-Sánchez, H. García-Mendoza et al., "Effect of the plasmid-DNA vaccination on macroscopic and microscopic damage caused by the experimental chronic Trypanosoma cruzi infection in the canine model," BioMed Research International, vol. 2013, 8 pages, 2013.

[23] O. Rodríguez-Morales, V. Monteón-Padilla, S. C. CarrilloSánchez et al., "Experimental vaccines against Chagas disease: a journey through history," Journal of Immunology Research, vol. 2015, Article ID 489758, 2015.

[24] J. Mota-Sánchez, "Vacunas de ADN: Inducción de la respuesta inmunitaria," Salud Publica de Mexico, vol. 51, pp. 463-469, 2009.

[25] B. Ferraro, M. P. Morrow, N. A. Hutnick, T. H. Shin, C. E. Lucke, and D. B. Weiner, "Clinical applications of DNA vaccines: current progress," Clinical Infectious Diseases, vol. 53, no. 3, pp. 296-302, 2011.

[26] A. Carabarin-lima, M. C. González-vázquez, L. Baylonpacheco, V. Tsutsumi, P. Talamás-rohana, and J. Luis, "Immunization with the recombinant surface protein $\mathrm{rTcSP} 2$ alone or fused to the CHP or ATPase domain of TcHSP70 induces protection against acute Trypanosoma cruzi infection," Journal of Vaccines \& Vaccination, vol. 1, pp. 1-10, 2010.

[27] P. C. Melby, B. Chandrasekar, W. Zhao, and J. E. Coe, "The hamster as a model of human visceral leishmaniasis: progressive disease and impaired generation of nitric oxide in the face of a prominent Th1-like cytokine response," The Journal of Immunology, vol. 166, no. 3, pp. 1912-1920, 2001.

[28] F. Tabatabaie, M. Mahdavi, S. Faezi et al., "Th1 platform immune responses against Leishmania major induced by thiol-specific antioxidant-based DNA vaccines," Jundishapur Journal of Microbiology, vol. 7, pp. 1-8, 2014.

[29] A. Krogh, È. Larsson, G. von Heijne, and E. L. L. Sonnhammer, "Predicting transmembrane protein topology with a hidden Markov model: application to complete genomes ", Journal of molecular biology, vol. 305, no. 3, pp. 567-580, 2001.

[30] K. D. Tsirigos, C. Peters, N. Shu, K. Lukas, and A. Elofsson, "The TOPCONS web server for consensus prediction of membrane protein topology and signal peptides," Nucleic Acids Research, vol. 43, pp. 401-407, 2015.

[31] K. Chou and H. Shen, "A new method for predicting the subcellular localization of eukaryotic proteins with both single and multiple sites: Euk-mPLoc 2.0," PLoS ONE, vol. 5, no. 4, article e9931, 2010.

[32] T. Goldberg, M. Hecht, T. Hamp et al., "LocTree3 prediction of localization," Nucleic acids research, vol. 42, pp. 350-355, 2014.

[33] J. J. Almagro Armenteros, C. K. Sønderby, S. K. Sønderby, H. Nielsen, and O. Winther, "DeepLoc : prediction of protein subcellular localization using deep learning," Bioinformatics, vol. 33, no. 21, pp. 3387-3395, 2017.

[34] C. Yu, Y. Chen, C. Lu, and J. Hwang, "Prediction of protein subcellular localization," Proteins: Structure, Function, and Bioinformatics, vol. 64, pp. 643-651, 2006.

[35] A. Campos-Neto, R. Porrozzi, K. Greeson et al., "Protection against cutaneous leishmaniasis induced by recombinant antigens in murine and nonhuman primate models of the human disease," Infection and immunity, vol. 69, no. 6, pp. 4103-4108, 2001.

[36] D. J. Shedlock and D. B. Weiner, "DNA vaccination: antigen presentation and the induction of immunity," Journal of Leukocyte Biology, vol. 68, no. 6, pp. 793-806, 2000.

[37] J. A. Wolff, J. J. Ludtke, G. Acsadi, P. Williams, and A. Jani, "Long-term persistence of plasmid DNA and foreign gone expression in mouse muscle," Human Molecular Genetics, vol. 1, no. 6, pp. 363-369, 1992.

[38] S. Gurunathan, C. Y. Wu, B. L. Freidag, and R. A. Seder, "DNA vaccines: a key for inducing long-term cellular immunity," Current Opinion in Immunology, vol. 12, no. 4, pp. 442-447, 2000.

[39] T. R. Smith, A. Patel, S. Ramos et al., "Immunogenicity of a DNA vaccine candidate for COVID-19," Nature Communications, vol. 11, pp. 1-13, 2020.

[40] O. Billaut-Mulot, T. Idziorek, M. Loyens, A. Capron, and G. M. Bahr, "Modulation of cellular and humoral immune responses to a multiepitopic HIV-1 DNA vaccine by interleukin-18 DNA immunization/viral protein boost," Vaccine, vol. 19, no. 20-22, pp. 2803-2811, 2001.

[41] C. Dye, "The leishmaniases in biology and medicine: edited by W. Peters and R. Killick- Kendrick. Academic Press, 1987. Volumes I and II, £105.00/US\$190.50. ISBN 012552101 4/0 12552102 2," Parasitology Today, vol. 4, no. 1, p. 28, 1988.

[42] B. Alemayehu and M. Alemayehu, "Leishmaniasis: a review on parasite, vector and reservoir host," Health Science Journal, vol. 11, no. 4, 2017.

[43] M. Wiese, O. Berger, Y. D. Stierhof, M. Wolfram, M. Fuchs, and P. Overath, "Gene cloning and cellular localization of a membrane-bound acid phosphatase of Leishmania mexicana," Molecular and Biochemical Parasitology, vol. 82, no. 2, pp. 153-165, 1996.

[44] M. Engstler, F. Weise, K. Bopp et al., "The membrane-bound histidine acid phosphatase TbMBAP1 is essential for endocytosis and membrane recycling in Trypanosoma brucei," Journal of Cell Science, vol. 118, no. 10, pp. 2105-2118, 2005.

[45] Y. Vanloubbeeck and D. E. Jones, “The immunology ofLeishmaniainfection and the implications for vaccine development," Annals of the New York Academy of Sciences, vol. 1026, no. 1, pp. 267-272, 2004.

[46] M. Chenik, H. Louzir, H. Ksontini, A. Dilou, I. Abdmouleh, and K. Dellagi, "Vaccination with the divergent portion of the protein histone $\mathrm{H} 2 \mathrm{~B}$ of Leishmania protects susceptible $\mathrm{BALB} / \mathrm{c}$ mice against a virulent challenge with Leishmania major," Vaccine, vol. 24, no. 14, pp. 2521-2529, 2006.

[47] P. C. Melby, G. B. Ogden, H. A. Flores et al., "Identification of vaccine candidates for experimental visceral leishmaniasis by immunization with sequential fractions of a cDNA expression library," Infection and Immunity, vol. 68, no. 10, pp. 55955602, 2000.

[48] P. A. Buffet, A. Sulahian, Y. J. Garin, N. Nassar, and F. Derouin, "Culture microtitration: a sensitive method for 
quantifying Leishmania infantum in tissues of infected mice," Antimicrobial agents and chemotherapy, vol. 39, no. 9, pp. 2167-2168, 1995.

[49] A. Schubach, F. Haddad, M. P. Neto et al., "Detection ofLeishmaniaDNA by polymerase chain reaction in scars of treated human patients," Journal of Infectious Diseases, vol. 178, no. 3, pp. 911-914, 1998.

[50] A. Thakur, H. Kaur, and S. Kaur, "Evaluation of the immunoprophylactic potential of a killed vaccine candidate in combination with different adjuvants against murine visceral leishmaniasis," Parasitology International, vol. 64, no. 1, pp. 70-78, 2015.

[51] M. R. Jaafari, A. Ghafarian, A. Farrokh-Gisour et al., "Immune response and protection assay of recombinant major surface glycoprotein of Leishmania (rgp63) reconstituted with liposomes in BALB/c mice," Vaccine, vol. 24, no. 29-30, pp. 5708-5717, 2006.

[52] C. Snapper and W. Paul, "Interferon-gamma and B cell stimulatory factor-1 reciprocally regulate Ig isotype production," Science, vol. 236, no. 4804, pp. 944-947, 1987.

[53] K. Anam, F. Afrin, D. Banerjee et al., "Differential decline in Leishmania membrane antigen-specific immunoglobulin $G$ (IgG), IgM, IgE, and IgG subclass antibodies in Indian KalaAzar patients after chemotherapy," Infection and immunity, vol. 67, no. 12, pp. 6663-6669, 1999.

[54] I. Benzel, F. Weise, and M. Wiese, "Deletion of the gene for the membrane-bound acid phosphatase of Leishmania mexicana," Molecular and Biochemical Parasitology, vol. 111, no. 1, pp. 77-86, 2000. 